\title{
COPD in China should receive more attraction
}

\author{
Guoqing Qian ${ }^{1,2 * \#}$, Fenying Yin ${ }^{1,2 *}$, Naibing Yang ${ }^{1,2}$ and Guoxiang $\operatorname{Li}^{1,2}$ \\ ${ }^{1}$ Department of General Internal Medicine, Ningbo First Hospital, School of Medicine, University of Ningbo, China \\ ${ }^{2}$ Department of Respiratory Medicine, Ningbo Hospital of Zhejiang University, P.R. China \\ ${ }^{\#}$ Guoqing Qian and Fenying Yin are co-first authors on this work
}

Chronic Obstructive Pulmonary Disease (COPD) is a leading cause of morbidity and mortality worldwide. China is not only the most populous country in the world ( 1.38 billion), but also the largest number of active smokers of the world (300 million adults) [1]. From 2004, there is just one national wide study has included a postbronchodilator spirometry examination [2]. That study reported that the prevalence of COPD in aged 40 years in China was $8.2 \%$ from 2002 to 2004 [3]. The prevalence and risk factors have been changed with the development of economy, population aging and ambient air pollution. Two articles which provide nationwide survey of the prevalence and assess the risk factors of COPD recently in The Lancet and The Lancet Respiratory Medicine [2,4].

In The Lancet, Chen Wang and colleagues reported that a crosssectional study in 2015 of 57,779 adults aged 20 years and through ten regions in China [4]. The prevalence of spirometry-defined COPD in China aged 20 years or older in 2015 was $8.6 \%$. It is the first time reported the prevalence of COPD among the aged 20-39 years in China and showed cigarette smoking, ambient air pollution, underweight, childhood chronic cough, parental history of respiratory diseases and low educations are major risk factors for COPD. The prevalence of COPD increased 67\% in 2015 (13.7\%) compared with 2004 (8.2\%) among the aged 40 years or older.

Smoking is one of the most important risk factors of COPD. That study reported that a two-fold elevate in the prevalence of COPD in 20-pack-years group. Interestingly, we find that women (18.5\%) had higher prevalence of COPD than men (15.0\%) when they had more than 20 pack-years of exposure. It is might note that the women could have susceptibility to cigarette smoke, which need further study. Passive smoking is associated with an increased prevalence of COPD and respiratory symptoms, which may have associated with 1.9 million excess deaths from COPD among never smokers in China [5]. Thus, smoking cessation efforts should be strengthened in China's public health policy.

In that study, $\mathrm{PM}_{25}$ was detected from the regional satelliteretrieved aerosol optical depth model, and the annual mean $\mathrm{PM}_{25}$ exposure is lower in rural [4]. And they found a high prevalence of COPD in never-smokers [4]. However, the prevalence of COPD is higher in rural than urban in entire population or never-smokers. We find that solid fuels (such as firewood, straw, and coal) still wildly used for cooking and heating in rural, which side effects of indoor air pollution and play a vital role in increasing prevalence of COPD in rural areas of China [6,7]. Therefore, we should pay more attention to indoor air pollution. What's more, the prevalence COPD is also related to economic and medical conditions in rural areas.
Spirometry is required to establish the diagnosis of COPD [8]. All COPD diagnosis by spirometry in that study [4]. Thus, spirometry should be promoted and be listed as one of the routine physical examination items in developed regions of China. On the other hand, there are shortage of spirometry and poor-quality control of spirometry in poverty areas. Dyspnoea, chronic cough or sputum production, and/or a history of exposure to risk factors for the disease should be considered COPD and be required to do spirometry [8]. This is of great value for areas that do not have spirometry and also answer the question of how to select the population for lung-function screening in China [9].

COPD as one of three non-communicable diseases in China, which poses a serious threat to the health of Chinese people. It is necessary to take the chance of health-care system transition as an opportunity to promote education, enhance lung-function screening and transfer disease treatment to health promotion and disease prevention in China. Only on this way, we would really achieve the healthy national goals in 2030 .

\section{References}

1. Sun Y, Sin DD (2018) Crisis of COPD in China: the future is now. Lancet Respir Med. [Crossref]

2. Fang L, Gao P, Bao H, Tang X, Wang B, et al. (2018) Chronic obstructive pulmonary disease in China: a nationwide prevalence study. Lancet Respir Med. [Crossref]

3. Zhong N, Wang C, Yao W, Chen P, Kang J, et al. (2007) Prevalence of chronic obstructive pulmonary disease in China: a large, population-based survey. Am J Respir Crit Care Med 176: 753-760. [Crossref]

4. Wang C, Xu J, Yang L, Xu Y, Zhang X, et al. (2018) Prevalence and risk factors of chronic obstructive pulmonary disease in China (the China Pulmonary Health [CPH] study): a national cross-sectional study. Lancet 391: 1706-1717. [Crossref]

5. Yin P, Jiang CQ, Cheng KK, Lam TH, Lam KH, et al. (2007) Passive smoking exposure and risk of COPD among adults in China: the Guangzhou Biobank Cohort Study. Lancet 370: 751-757. [Crossref]

6. Chen T, Liao H (2018) The Disease Burden of Indoor Air Pollution from Solid Fuel Use in China. Asia Pac J Public Health. [Crossref]

7. Assad NA, Kapoor V, Sood A (2016) Biomass smoke exposure and chronic lung disease. Curr Opin Pulm Med 22: 150-157. [Crossref]

${ }^{\star}$ Correspondence to: Guoqing Qian, Department of General Internal Medicine, Ningbo First Hospital, No.59 Liuting Street, Haishu District, Ningbo City, Zhejiang Province, China 315010, Tel: 86-574-8708-9874, E-mail: bill.qian@ outlook.com

Key words: $C O P D$, prevalence, risk factors

Received: April 17, 2018; Accepted: May 15, 2018; Published: May 18, 2018 
8. The Global Initiative for Chronic Obstructive Lung Disease. Global strategy for the diagnosis, management, and prevention of chronic obstructive pulmonary disease (Updated 2018).
9. Qian G, Ying F, Li G (2017) Tiotropium in Early-Stage COPD. $N$ Engl J Med 377: 2292-2293. [Crossref]

Copyright: (C2018 Qian G. This is an open-access article distributed under the terms of the Creative Commons Attribution License, which permits unrestricted use, distribution, and reproduction in any medium, provided the original author and source are credited. 